\title{
Comparación histomorfométrica in vitro del ligamento periodontal de premolares extraídos mantenidos en cuatro medios de conservación
}

\author{
In vitro histomorphometric comparison of periodontal ligament of extracted \\ premolar teeth preserved in different media storage
}

\author{
PROIKOPOWITSCH I* \\ CABRALES SALGADO R** \\ DÍAZ CABALLERO $\mathrm{A} * * *$ \\ SIMANCAS PALLARES M $* * * *$
}

\begin{abstract}
Prokopowitsch I, Cabrales Salgado R, Díaz Caballero A, Simancas Pallares M. Comparación histomorfométrica in vitro del ligamento periodontal de premolares extraídos mantenidos en cuatro medios de conservación. Av Periodon Implantol. 2013; 25, 1: 41-47.
\end{abstract}

\begin{abstract}
RESUIMEN
Objetivo: El propósito de este estudio fue evaluar histológica y morfométricamente los resultados de diferentes tipos de medios de almacenamiento para los dientes avulsionados en el mantenimiento de la preservación de la integridad del ligamento periodontal.

Material y métodos: Estudio de tipo experimental de laboratorio, se utilizaron veintitrés premolares extraídos por motivos ortodóncicos. Los medios de conservación evaluados fueron: leche tipo "B"y tipo "C", solución salina y medio seco. Un total de cinco dientes fueron almacenados en cada uno de los medios de conservación durante 120 minutos. Otros tres dientes sirvieron como controles fijados inmediatamente después de la extracción representando el ligamento periodontal íntegro. Los dientes se fijaron, procesaron y tiñeron con hematoxilina y eosina para evaluación histológica a través de microscopia óptica.

Resultados: La edad de los pacientes que aportaron los dientes osciló entre 13 a 17 años. Los resultados de la evaluación cualitativa mostraron que la solución fisiológica fue el medio de almacenamiento más adecuado seguido de la leche tipo $\mathrm{C}$ y tipo B. En el análisis estadístico no hubo diferencia estadísticamente significativa entre los grupos de solución fisiológica y leche tipo C. Después de 120 minutos se encontraron diferencias estadísticamente significativas entre las alteraciones histomorfométricas del grupo control y los grupos de dientes que se mantuvieron en condiciones de humedad y los secos.

Conclusión: Dados los resultados de este estudio, la solución salina (grupo II) y la leche tipo C (Grupo III) pueden considerarse como las formas de conservación más adecuadas de los dientes avulsionados.
\end{abstract}

PALABRAS CLAVE: Periodoncia, heridas y traumatismos, procesos alveolares, avulsión de diente, ligamento periodontal (DeCS Bireme).

\section{SUMMMARY}

Objective: The aim of this study was to histologically and morphometrically evaluate the results of different types of storage media for avulsed teeth in the maintenance and preserving the integrity of the periodontal ligament.

* $\quad$ DDS, Ms, Ph.D. Endodoncia. Universidad de São Paulo Brasil. Profesor Titular Universidad Cruzeiro do Sul. São Paulo. Brasil.

** DDS, Ph.D. Endodoncia. Universidad de São Paulo Brasil. Profesor invitado Facultad de odontología. Universidad de Cartagena. Colombia.

*** Odontólogo. Especialista en Periodoncia. Estudiante de Doctorado en Ciencias Biomédicas. Profesor Titular Facultad de Odontología. Universidad de Cartagena. Colombia.

**** Odontólogo. Estudiante de Maestría en Epidemiología Clínica. Integrante: grupo interdisciplinario de investigaciones y tratamientos odontológicos (GITOUC). Facultad de Odontología. Universidad de Cartagena. 
Material and methods: Experimental study. It was used twenty-three extracted premolars for orthodontic reasons. Preserving methods evaluated were: type " $B$ " and " $C$ " milk, saline and dry environment. A total of five teeth were stored in each storage media for 120 minutes. Three teeth served as controls fixed immediately after extraction representing the entire periodontal ligament. The teeth were fixed, processed and stained with hematoxylin/eosin for histological evaluation by light microscopy.

Results: The age of the patients who provided the teeth ranged from 13-17 years. The results of the qualitative assessment showed that saline was the most appropriate storage medium, followed by milk type $\mathrm{C}$ and type $\mathrm{B}$. In the statistical analysis there was no statistically significant difference between saline and type $\mathrm{C}$ milk. After 120 minutes it was found statistically significant differences between histomorphometric changes in the control group and groups of teeth that were kept in wet conditions and dry.

Conclusion: Given the results of this study, saline (group II) and milk type C (Group III) can be considered as the most suitable form of storage of avulsed teeth.

KEY WORDS: Periodontics, tooth Injuries, tooth avulsion, alveolar process, periodontal ligament (MeSH Database).

Fecha de recepción: Noviembre de 2010.

Fecha de aceptación: Enero de 2011.

\section{INTRODUCCIÓN}

Los dientes permanentes avulsionados constituyen una de las formas más graves de trauma dentoalveolar, dado que el diente está completamente desplazado de su alveolo y puede causar daños a las estructuras periodontales, incluso ruptura de las fibras del ligamento periodontal (LP) y cemento. El reimplante inmediato de un diente avulsionado es una de las mejores alternativas de tratamiento, ya que puede restaurar, al menos temporalmente, la estética y función de la región traumatizada (1). Una vez que el diente es reimplantado, hay una mejor oportunidad de cicatrización del LP (2). Sin embargo, la experiencia clínica demuestra que la mayoría de los dientes avulsionados sólo son reimplantados después de un largo tiempo fuera del alveolo por lo general en modo de conservación seco o en pobres condiciones de almacenamiento $(3,4)$. Dependiendo del tiempo que transcurre fuera del alveolo y del medio de almacenamiento, pueden ocurrir diversos fenómenos tales como necrosis pulpar, degeneración de las fibras LP y cementoblastomas. La principal causa de fracaso de un reimplante la origina la reabsorción radicular inflamatoria la cual es originada por restos necróticos de fibras del LP sobre la raíz $(2,5)$.

Un medio húmedo de almacenamiento de los dientes avulsionados puede controlar los efectos nocivos de la desecación durante un período determinado, que puede ser aún más importante para la reimplantación del diente que el período extraoral $(2,6)$. Algunas de las características del medio de almacenamiento, tales como pH 7 y la osmolaridad, deben ser compatibles con la supervivencia de las células (8). Idealmente, los medios de almacenamiento deben estar libres de bacterias y contaminantes (6). Se demostró en diversos ensayos que las células presentan mayor supervivencia y proliferación cuando los dientes se mantienen en un medio refrigerado en comparación con los medios utilizados a temperatura ambiente $(9,10)$. Algunas soluciones que sean fácilmente accesibles al momento de un accidente, se evalúan como medio de almacenamiento para los dientes avulsionados. Debido a su baja osmolaridad, el agua de grifo demostró que produce resultados similares a los de los medios de almacenamiento secos (2).

A pesar de su osmolaridad adecuada, la solución salina es capaz de mantener la vitalidad celular del LP sólo por un corto período (11-17). La saliva es un mejor medio de almacenamiento provisional para dientes avulsionados que el agua del grifo, pero tiene menor osmolaridad que la leche y puede presentar productos bacterianos (8-18). La leche bovina presenta hasta ahora los mejores resultados entre los medios de almacenamiento que se encuentran más comúnmente en el sitio de un accidente. Sumado a lo anterior, se considera un método de preservación económico, de fácil acceso tanto en países desarrollados como en vía 
de desarrollo. De igual forma sus características bioquímicas ( $\mathrm{pH}$, contenido de aminoácidos, vitaminas, proteínas, pasteurización) le confieren propiedades altamente nutritivas y de tal forma, uno de los métodos más adecuados para la preservación de un diente avulsionado $(12,14,17)$.

La solución salina de Hank balanceada, demuestra resultados más favorables que la leche para la conservación de dientes avulsionados (13). Viaspan (Belzer UW, CSS, Du Pont Farmacéutica, Wilmington, DE, EE.UU.), es una solución desarrollada originalmente para el preservación de órganos para trasplantes, la cual, presenta resultados favorables en las primeras 12 horas de almacenamiento $(13,14)$. La solución de Euro-Collins se desarrolló como una modificación de la original solución de Collins y se utiliza para preservación hipotérmica de órganos recogidos para trasplante $(15,16)$.

Con la anterior evidencia científica disponible se pone en consideración la necesidad de evidencia sobre medios de conservación en dientes extraídos y evaluados histomorfométricamente como aporte para la toma de decisiones clínicas.

El propósito del presente estudio fue evaluar microscópica e histomorfométrica el ligamento periodontal de dientes humanos, mantenidos en diferentes medios de conservación post extracción; incluyendo dos tipos de leche con propiedades fisiológicas y bioquímicas diferentes las cuales inducen diferentes tipos de respuesta en los tejidos orales.

\section{MATERIAL Y MÉTODOS}

Estudio de tipo experimental de laboratorio, donde se utilizaron 23 dientes humanos (premolares superiores e inferiores), extraídos por motivos ortodóncicos de pacientes con edades entre 13-17 años. Las extracciones fueron realizadas de manera atraumática. Después de la extracción todos los dientes se lavaron con solución salina y luego a cada diente se le asignó un código de tres (03) dígitos y posteriormente fueron incluidos en una base de datos en Microsoft ${ }^{\circledR}$ Excel versión 2007. Para dividir aleatoriamente los dientes a cada grupo de tratamiento se utilizó el programa Microsoft $₫$ Excel versión 2007. Se dividieron los dientes cinco grupos, a cada tipo de tratamiento, como sigue:

- Grupo control o grupo I: Tres dientes, que después de la extracción, se fijaron en formol al 10\%.
- Los grupos experimentales: Con un número de cinco dientes cada uno. Los cuatro grupos experimentales se mantuvieron durante 120 minutos sobre las diferentes formas de conservación:

- Grupo II: Solución salina al 0,9\% (Baxter Health Care Corporation. One Baxter Parkway Deerfield, IL 60015-4625) 4-10 C.

- Grupo III: Leche pasteurizada de tipo C* (Planta de Procesamiento de Productos Lácteos de la Cooperativa Ltda. Alta Paulista. Tupa, São Paulo), con una temperatura inicial entre $4-10^{\circ} \mathrm{C}$, llegando gradualmente a la temperatura ambiente. Tipo de leche que después del proceso de pasteurización tiene un contenido de microorganismos de aproximadamente $300.000 \mathrm{ml}$ de leche (antes de la pasteurización) y tolerancia a coliformes de 0,2 ml. Puede sufrir dos tipo de pasteurización (lenta y rápida).

- Grupo IV: Leche pasteurizada Tipo B* (Planta de Procesamiento de Productos Lácteos de la Cooperativa Ltda. Alta Paulista. Tupa, São Paulo), con una temperatura inicial entre 4 y $10^{\circ} \mathrm{C}$, llegando gradualmente a la temperatura ambiente. Este tipo de leche se diferencia de la tipo $\mathrm{C}$ porque: tiene almacenamiento más sofisticado, la extracción es de manera mecánica, sin embargo el proceso de transporte la hace susceptible de contaminarse. El contenido de microorganismos es de aproximadamente $500.000 \mathrm{ml}$ antes de la pasteurización y $30.000 \mathrm{ml}$ después de la pasteurización; su tolerancia a coliformes es de aproximadamente $0,5 \mathrm{ml}$.

- GrupoV: Medio seco sobre una gasa estéril.

Tras el período de almacenamiento previsto (120 minutos en cada medio) los dientes se acondicionaron en frascos de vidrios y fijados en formaldehído al $10 \%$. Finalizado el proceso de descalcificación con ácido fórmico al $40 \%$ haciendo recambio cada $48 \mathrm{~h}$, los dientes se cortaron transversalmente en dos partes iguales (cervical y apical) y luego deshidratados con EDTA, aclarados e incluidos en parafina. Se obtuvieron, a continuación, 20 secciones semiseriadas en el sentido longitudinal de cada muestra y de seis micrómetros de espesor. Las preparaciones fueron teñidas a través de la técnica de hematoxilina de Harris y eosina a $1 \%$, de acuerdo a los procedimientos de laboratorio de rutina.

Se realizó un análisis cualitativo de las células y las fibras del ligamento periodontal, de acuerdo con una evaluación de tres examinadores previamente calibrados y cegados a cada tipo de tratamiento, a tra- 
vés de microscopía óptica (Microscopio óptico NIKON E200). Se consideraron parámetros histomorfológicos de la preservación del tejido del ligamento periodontal teniendo en cuenta las siguientes características: organización de fibra, grado de degeneración celular, forma, tamaño y coloración de los núcleos celulares. En esta evaluación se asignaron códigos entre 1 y 4 para clasificar los medios de conservación como: pobres (1), regular (2), bueno (3), excelente (4).

\section{Análisis estadístico}

Se realizaron pruebas de estadística descriptiva. Para las variables cuantitativas se reportó media y desviación estándar, para las variables cualitativas se reportó frecuencias y proporciones. Para la realización de pruebas de estadística inferencial se comprobó la normalidad de la distribución de los datos. Dadas estas condiciones se realizó prueba de Kruskall-Wallis. Nivel de confianza del estudio: 95\%. Error tipo I: 5\%. El análisis estadístico se realizó con el paquete Stata ${ }^{\mathrm{TM}}$ versión 9.0 (4905 Lakeway Drive - College Station, Texas 77845 USA).

\section{Consideraciones éticas}

Se tuvieron en cuenta las consideraciones éticas establecidas en el Informe de Helsinki, para la experimentación con órganos y tejidos obtenidos de seres humanos. Se mantuvo discreción con la identidad de los participantes, a los cuales se les solicitó la firma de un consentimiento informado escrito a sus acudientes o padres.

\section{RESULTADOS}

De acuerdo a los parámetros utilizados morfológicos e histomorfométricos, los diferentes grupos mostraron las siguientes características:

- Grupo I (control): Los especímenes presentaron fibras longitudinales dispuestas en columnas organizadas. Las características celulares fueron normales, se observaron fibroblastos maduros. Estos aspectos caracterizan en gran medida la preservación de estas estructuras (Figura $1 \mathrm{~A}$ ).

- Grupo II (solución salina): Mostró, tanto en relación con las fibras, como en las características celulares, propiedades similares a las observadas en el grupo control (Grupo I).
- Grupo III (leche tipo C*): Mostraron características normales de células, como los grupos I y II. En relación con el colágeno, cabe señalar que éste, a pesar de tener algún grado de degeneración, aún conservaba una disposición en forma de paquetes.

- Grupo IV (leche de tipo B*): Presentó células dentro de los límites normales (fibroblastos maduros), la disposición de las fibras de colágeno no se presentó en forma de bloques organizados como los que se observaron en los grupos (I, II y III) y mostró, en vez de esto, un aspecto homogéneo y con menor intensidad de tinción.

- Grupo V (conservación "en seco"): Se presentó el mayor compromiso de los componentes del ligamento periodontal, tanto en relación a las células y fibras. Los núcleos fueron los más intensamente teñidos, y algunos picnóticos. Con respecto a las fibras, se pudo observar las zonas más extensas de hialinización, privando a la organización de cursos de agua, en contraste con la del grupo control o Grupo I (Figura 1B).

En el análisis bivariado entre grupos se muestran los promedios de las posiciones de las muestras mediante análisis de Kruskall-Wallis (Tabla 1). Se observa que la diferencia entre el grupo control (grupo I, Figura $1 A)$, donde los dientes fueron extraídos y fijados en

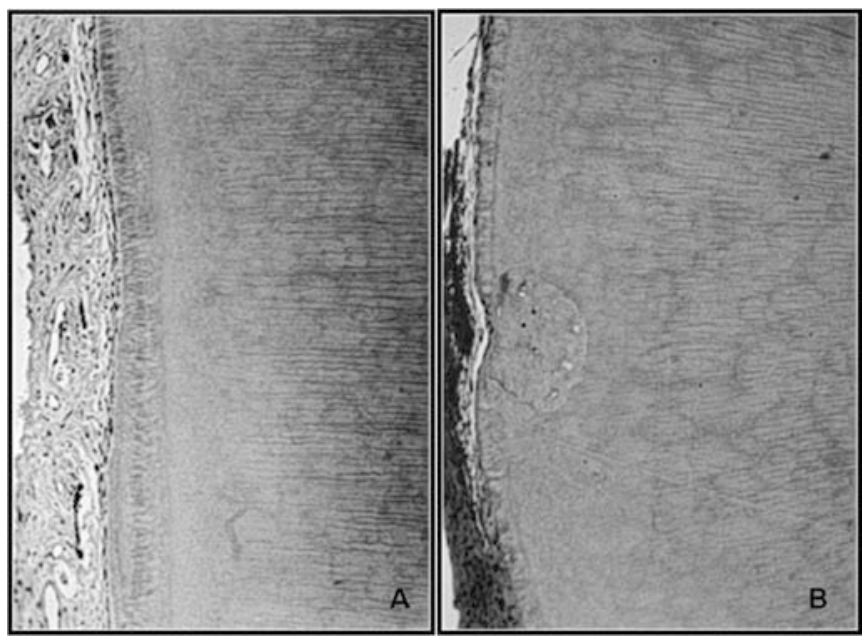

Fig.1: A. Imagen microscópica de las fibras del ligamento periodontal después de la extracción, mostrando fibras longitudinales constituidas en columnas. Células con características uniformes y presencia de fibroblastos maduros, caracterizando una gran preservación del ligamento periodontal. B: imagen microscópica de las fibras del ligamento periodontal conservado en medio seco, mostrando un colapso de las células y extensas zonas de hialinización de las fibras del ligamento periodontal, núcleos intensamente teñidos y algunos picnóticos. 


\begin{tabular}{|c|c|c|}
\hline \multicolumn{3}{|c|}{$\begin{array}{c}\text { TABLA 1.- COMPARACIONES ENTRE } \\
\text { LAS POSICIONES MEDIAS DE LAS } \\
\text { MUESTRAS DE LOS DIFERENTES } \\
\text { GRUPOS RELATIVAS A LA EVALUACIÓN } \\
\text { CUALITATIVA DE LAS CÉLULAS DEL } \\
\text { LIGAMENTO PERIODONTAL (Y FIBRA) }\end{array}$} \\
\hline $\begin{array}{l}\text { Grupos } \\
\text { comparados }\end{array}$ & $\begin{array}{l}\text { Diferencia } \\
\text { entre } \\
\text { las medias }\end{array}$ & $\begin{array}{l}\text { Valor } \\
\text { de } p\end{array}$ \\
\hline $\begin{array}{l}\text { Control vs sol. fisiológica } \\
\text { Control vs leche C } \\
\text { Control vs leche B } \\
\text { Control vs en seco } \\
\text { Sol. fisiológica vs leche C } \\
\text { Sol. fisiológica vs leche B } \\
\text { Sol. fisiológica VS en seco } \\
\text { Leche C vs leche B } \\
\text { Leche C vs en seco } \\
\text { Leche B vs en seco }\end{array}$ & $\begin{array}{r}3,00 \\
4,50 \\
8,50 \\
11,50 \\
1,50 \\
5,50 \\
8,50 \\
4,00 \\
7,00 \\
3,00\end{array}$ & $\begin{array}{l}0,05^{*} \\
0,001^{*} \\
0,001^{*} \\
0,001^{*} \\
\text { N.S. } \\
0,001^{*} \\
0,001^{*} \\
0,01 * \\
0,001^{*} \\
0,05^{*}\end{array}$ \\
\hline
\end{tabular}

formaldehído al 10\%, caracterizando condiciones similares a las de un reimplante inmediato, y el grupo II fueron menores $(p=0,05)$ que las observadas entre los grupos I y III y I y IV $(p=0,001)$. También en relación con la tabla 1, la diferencia entre los grupos $\mathrm{V}$ (dientes mantenidos en seco, Figura 1B) y Grupo II (solución salina) resultó estadísticamente significativa $(p=0,001)$, al igual que entre los grupos V y IV (leche tipo B) $(p=0,05)$. Es importante recalcar que, la diferencia entre los grupos V (seco) y III (leche tipo C) resultó estadísticamente significativa $(p=0,001)$.

\section{DISCUSIÓN}

La avulsión, consecuencia de un trauma dentoalveolar, es uno de los problemas que causan mayor preocupación para los padres y niños, especialmente cuando los dientes que están implicados son permanentes (2).

Diversos autores sugieren que, en caso de avulsión dental, se debe realizar reimplante en el menor tiempo posible, aunque las condiciones no fueran ideales. Sin embargo, en tales circunstancias, esta conducta debe ser vista sólo como una posibilidad $(1,8)$. Hay muchas variables que pueden influir en el pronóstico de un diente reimplantado. Algunas como el tiempo transcurrido desde la avulsión hasta el momento de la reimplantación y el medio de conservación después de la avulsión dental, son claves para el éxito del reimplante y por lo general no están al alcance del odontólogo general (2-6).

De acuerdo a la metodología utilizada en el presente estudio, los resultados difieren de otros resultados de investigaciones in vitro con respecto a las condiciones de mantenimiento de la leche en relación con el suero fisiológico (11-17) para dientes reimplantados. Estos autores reportan que la leche contiene importantes nutrientes, tales como aminoácidos, carbohidratos y vitaminas los cuales reducen la actividad enzimática de las células del ligamento periodontal, situación contraria a los dientes almacenados en solución salina. Además, se conoce que el proceso de pasteurización es capaz de inactivar enzimas potencialmente dañinas para el ligamento periodontal. De manera similar a los resultados obtenidos, están los resultados de Andreassen, 1994 (1); Patil, Dumska, Sydiskis, en 1994 (19); Pileggi y cols., en 2002 (17), quienes concluyen que la leche es un medio favorable de conservación para los dientes avulsionados.

Blömlof y Hammarström, en 1981 (19), estudiaron la osmolaridad de diferentes medios de almacenamiento, encontrando que la leche y solución salina se mostraron muy similares. En la presente investigación se encontró que no hubo diferencia estadísticamente significativa entre los Grupos II (solución salina) y III (leche tipo C). También, de acuerdo a la tabla I, se encontró que existen diferencias estadísticamente significativas entre todos los grupos de dientes que se mantuvieron en medio húmedo (solución salina y la leche de tipo B y tipo C) y las que mantienen secas. Estos hallazgos son consistentes con los reportados en los trabajos de Söder y cols, en 1977 (22); Oswald, Harrington y Hassel, en 1980 (23); Layug y cols, en 1998 (21), los cuales consideran que el efecto negativo de la exposición del diente con el medio ambiente está directamente relacionada con la disminución progresiva de la vitalidad ligamento periodontal.

Andreassen, en 1988 (20), reportó la existencia de una capa vital de las células del ligamento periodontal que se relaciona directamente con la conservación de entorno fisiológico, lo que favorece la protección contra la reabsorción radicular.

Cabe señalar que el mantenimiento de un ligamento periodontal viable es esencial para la reparación "ad integrum" de la inserción periodontal de los dientes 
reimplantados tal como lo mencionan Oswald y cols., en 1980 (23); Emerich, en 2010 (24). De esta manera, los resultados obtenidos permiten establecer que se puede considerar que la calidad de las células del ligamento periodontal de dientes extraídos es superior al almacenarlos en condiciones de humedad al comparar con el almacenamiento en forma seca, que está de acuerdo con los informes de Andreasen, 1981 (2); Blomlöf y cols., 1983 (12); Andreasen, en 1994 (1) y Krasner (26), proporcionando un mejor pronóstico en la expectativa de vida del diente reimplantado.

A pesar del gran número de estudios que ponen de manifiesto la superioridad de la leche como un medio de almacenamiento, hay pocos reportes en la literatura que tienen en cuenta la influencia de los diferentes componentes de la leche en la preservación del ligamento periodontal de dientes extraídos y su evaluación histomorfométrica. Según Blomlöf, Hammarstrom Otteskog, en 1981 (7), reportaron que las diferencias en calidad de la leche, en cuanto al contenido de materia grasa, no tiene ninguna influencia en la preservación de células del ligamento periodontal. Sumado a esto, Sigalas y cols., en 2004 (28), demuestran que la leche baja en grasa podría servir como una alternativa si hay hielo que ayude a atemperar su condición. Por otra parte, en 1983, Blomlöf y cols. (27) consideran que la composición de la leche puede variar en diferentes partes del mundo, que puede ser aconsejable estimar su composición, así como su osmolaridad, antes de ser recomendado para su uso clínico.

Frente a un análisis global de los resultados contenidos en este trabajo, se hace hincapié en que, en caso de avulsión dental donde no es posible el reimplante de inmediato, se sugiere mantener el diente en condiciones de humedad, para una mejor preservación de las fibras del ligamento periodontal.

\section{CONCLUSIONES}

Dados los hallazgos y en las condiciones experimentales establecidas, parece razonable concluir que después de 120 minutos se encontraron diferencias significativas entre el grupo control y los grupos de dientes que se mantuvieron en condiciones de humedad (leche y solución salina) y los que se quedaron secos.

En cuanto al medio de transporte y conservación utilizado, los dientes en el grupo II (solución salina) tuvieron resultados más favorables en cuanto a la preservación del ligamento periodontal.
La solución salina (grupo II) y la leche tipo C (grupo III) puede considerarse como formas de conservación de los dientes avulsionados; sin embargo se necesitan estudios in vivo que comparen estos tipos de medios de conservación. La leche de tipo B muestra los resultados menos favorables, como medio de mantener los dientes avulsionados en condiciones in vitro.

\section{BIBLOGRAFÍA}

1. Rosenblatt $A$. The best treatment for avulsed permanent teeth. Evid Based Dent 2010;1 1:42-3.

2. Andreasen JO. Effect of extra-alveolar period and storage media upon periodontal and pulpal healing after replantation of mature permanent incisors in monkeys. Int J Oral Surg 1981;10:43-53.

3. Guedes OA, de Alencar AH, Lopes LG, Pécora JD, Estrela C. A retrospective study of traumatic dental injuries in a Brazilian dental urgency service. Braz Dent J 2010;21: 153-7.

4. Andreasen JO, Borum MK, Jacobsen HL, Andreasen FM. Replantation of 400 avulsed permanent incisors. IV. Factor related to periodontal ligament healing. Endod Dent Traumatol 1995;11:76-89.

5. Hammarström L, Blomlöf L, Feiglin B, Andersson L, Lindskog S. Replantation of teeth and antibiotic treatment. Endod Dent Traumatol 1986;2:51-7.

6. Blomlöf L. Storage of human periodontal ligament cells in a combination of different media. J Dent Res 1981; 60:1904-6.

7. Blomlöf L, Otteskog P, Hammarström L. Effect of storage in media with different ion strengths and osmolalities on human periodontal ligament cells. Scand J Dent Res 1981;89:180-7.

8. Lindskog S, Blomlöf L. Influence of osmolality and composition of some storage media on human periodontal ligament cells. Acta Odontol Scand 1982;40:43541.

9. Lekic P, Kenny D, Moe HK, Barretti E, McCulloch CA. Relationship of clonogenic capacity to plating efficiency and vital dye staining of human periodontal ligament cells: Implications for tooth replantation. J Periodontal Res 1996;31:294-300. 
10. Schwartz O, Andreasen FM, Andreasen JO. Effects of temperature, storage time and media on periodontal and pulpal healing after replantation of incisors in monkeys. Dent Traumatol 2002;18:190-5.

11. Andreasen JO, Schwartz O. The effect of saline storage before replantation upon dry damage of the periodontal ligament. Endod Dent Traumatol 1986;2:67-70.

12. Blomlöf L, Lindskog S, Hammarström L. Periodontal healing of exarticulated monkey teeth stored in milk or saliva. Scand J Dent Res 1981;89:251-9.

13. Hiltz J, Trope M.Vitality of human lip fibroblasts in milk, Hanks balanced salt solution and Viaspan storage media. Endod Dent Traumatol 1991;7:69-72.

14. Trope M, Friedman S. Periodontal healing of replanted dog teeth stored in Viaspan, milk and Hank's balanced salt solution. Endod Dent Traumatol 1992;8:183-8.

15. Hrabalová M, Bachleda P, Lubuská L, Kojecký Z, Zadrazil J, Krejcí K, Al Jabry S. Effect of various protective solutions on function after kidney transplantation. Biomed Pap Med Fac Univ Palacky Olomouc Czech Repub 2003;147:197-202.

16. Jassem W, Armeni T, Quiles JL, Bompadre S, Principato G, Battino M. Protection of mitochondria during cold storage of liver and following transplantation: comparison of the two solutions, University of Wisconsin and EuroCollins. J Bioenerg Biomembr 2006;38:49-55.

17. Pileggi R, Dumsha TC, Nor JE. Assessment of posttraumatic PDL cells viability by a novel collagenase assay. Dent Traumatol 2002;18:186-9.

18. Ozan F, Polat ZA, Tepe B, Er K. Influence of storage media containing Salvia officinalis on survival of periodontal ligament cells. J Contemp Dent Pract 2008;9:17-24.

19. Patel S, Dumsha TC, Sydiskis RJ. Determining periodontal ligament (PDL) cell vitality from exarticulated teeth stored in saline or milk using fluorescein diacetate. Int Endod J 1994;27:1-5.

20. Sottovia AD, Sottovia Filho D, Poi WR, Panzarini SR, Luize DS, Sonoda CK. Tooth replantation after use of EuroCollins solution or bovine milk as storage medium: a histomorphometric analysis in dogs. J Oral Maxillofac Surg 2010;68:111-9.

21. Layug ML, Barrett EJ, Kenny DJ. Interim storage of avulsed permanent teeth. J Can Dent Assoc 1998;64:35763.

22. Söder PO, Otteskog P, Andreasen JO, Modéer T. Effect of drying on viability of periodontal membrane. Scand J Dent Res 1977;85:164-8.

23. Oswald RJ, Harrington GW, Van Hassel HJ. A postreplantation evaluation of air-dried and saliva stored avulsed teeth. J Endod 1980;6:546-51.

24. Emerich K, Czerwinska M, Ordyniec-Kwasnica I. Immediate self-replantation of an avulsed permanent mandibular incisor - a case report. Dent Traumatol 2010; 26:351-4

25. Andreasen JO, Borum MK, Jacobsen HL, Andreasen FM. Replantation of 400 avulsed permanent incisors. 4. Factors related to periodontal ligament healing. Endod Dent Traumatol 1995;1 1:76-89.

26. Krasner P. Modern treatment of avulsed teeth by emergency physicians. Am J Emerg Med 1994;12:2416.

27. Blomlöf L, Lindskog S, Andersson L, Hedström KG, Hammarström L. Storage of experimentally avulsed teeth in milk prior to replantation. J Dent Res 1983;62: 912-6.

28. Sigalas E, Regan JD, Kramer PR, Witherspoon DE, Opperman LA. Survival of human periodontal ligament cells in media proposed for transport of avulsed teeth. Dent Traumatol. 2004;20:21-8.

\section{CORRESPONDENCIA}

Miguel Ángel Simancas

Facultad de Odontología. Universidad de Cartagena Campus de Ciencias de La Salud

Barrio Zaragocilla

Cartagena de Indias. Bolívar. Colombia

Correo electrónico: msimancasp@gmail.com 